\title{
DNA barcode-based delimitation of the Glossiphonia species
}

\author{
Bolbat N. ${ }^{1 *}$, Bolbat A. ${ }^{2}$, Kaygorodova I. ${ }^{1,2}$ \\ ${ }^{1}$ Irkutsk State University, Irkutsk, Russia \\ ${ }^{2}$ Limnological Institute, SB RAS, Irkutsk, Russia \\ *e-mail:mandzyak@lin.irk.ru
}

Key words: species delimitation, molecular taxonomy, Glossiphonia, Eastern Siberia

Motivation and Aim: Our knowledge about global hirudofauna is getting continuously enriched, however the taxonomic uncertainty of the Glossiphonia genus still persists. To resolve the issues associated with the determination of species status, molecular and bioinformational methods must be integrated into the practice of taxonomic research along with classical analysis of morphological features. In present study we utilized a combination of different approaches to solve the problem of leech species delimitation within the Glossiphonia genus.

Methods and Algorithms: Morphological analysis was carried out in accordance with the existent classification [1,2]. Molecular analysis was performed by the standard phenol-free extraction and the subsequent amplification with universal COI primers. Phylogenetic analysis was performed with the use of Maximum Likelihood (ML) method implemented in MEGA 7.0 [3], to determine the threshold value of intraspecific genetic variations we used GMYC method based on the analysts of ultrametric tree obtained from Bayesian inference (BI) in BEAST v.1.8.4 [4]. Additionally, ABGD program was used to determine the barcode gap [5].

Results: The results of morphological, molecular and 4 bioinformational methods (ML, $\mathrm{BI}, \mathrm{GMYC}$ и ABGD) indicate the independent taxonomic status of at least eight species within the Glossiphonia genus, including two Eastern Siberian species - G. verrucata and Glossiphonia sp.

Conclusion: The combination of results of present study points out the increased objectivity of biodiversity estimation within the Glossiphonia leech genus in case of implementing classical methods in conjunction with molecular, phylogenetic and bioinformational methods of analysis.

Acknowledgements: The study was performed in frame of the state assignment No. 03452016-0002 (AAAA-A16-116122110066-1), and in part of the Baikal fauna investigation was financially supported by the Russian Foundation for Basic Research (RFBR), project No. 17-2900097.

\section{References}

1. Lukin E.I. Leech of fresh and brackish waters. Fauna of the USSR. Leningrad: Science Publ., 1976.

2. Nesemann H., Neubert E. Clitellata, Branchiobdellada, Acanthobdellada, Hirudinea. J. Schwoebel and P. Zwig (eds.). Susswasser fauna von Mitteleuropa. 1999;6(2):1-178.

3. Kumar S., Stecher G., Tamura K. MEGA7: Molecular evolutionary genetics analysis version 7.0 for bigger datasets. Mol. Biol. Evol. 2016;33(7):1870-1874.

4. Drummond A.J., Suchard M.A., Xie D., Rambaut A. Bayesian phylogenetics with BEAUti and the BEAST 1.7. Mol. Biol. Evol. 2012;29:1969-1973.

5. Puillandre N, Lambert A, Brouillet S, Achaz G. ABGD, Automatic Barcode Gap Discovery for primary species delimitation. Mol. Ecol. 2012;21:1864-1877. 